University of Nebraska - Lincoln

DigitalCommons@University of Nebraska - Lincoln

6-2009

\title{
Price Trends and Patterns in Technical Analysis: A Theoretical and Empirical Examination
}

\author{
Geoffrey C. Friesen \\ University of Nebraska-Lincoln, gfriesen2@unl.edu \\ Paul Weller \\ University of lowa, Iowa City, IA, Paul-Weller@uiowa.edu \\ Lee Dunham \\ Creighton University, Omaha, NE, leedunham@creighton.edu
}

Follow this and additional works at: https://digitalcommons.unl.edu/financefacpub

Part of the Finance and Financial Management Commons

Friesen, Geoffrey C.; Weller, Paul; and Dunham, Lee, "Price Trends and Patterns in Technical Analysis: A Theoretical and Empirical Examination" (2009). Finance Department Faculty Publications. 11.

https://digitalcommons.unl.edu/financefacpub/11

This Article is brought to you for free and open access by the Finance Department at DigitalCommons@University of Nebraska - Lincoln. It has been accepted for inclusion in Finance Department Faculty Publications by an authorized administrator of DigitalCommons@University of Nebraska - Lincoln. 


\title{
Price trends and patterns in technical analysis: A theoretical and empirical examination
}

\author{
Geoffrey C. Friesen,* Paul A. Weller, ${ }^{* *}$ and Lee M. Dunham *** \\ * Department of Finance, College of Business, University of Nebraska-Lincoln, Lincoln, NE 68508, USA \\ (Corresponding author: tel 402 472-2330, fax 402 472-5440, email gfriesen2@unl.edu) \\ ** Department of Finance, Henry B. Tippie College of Business, University of Iowa, \\ Iowa City, IA 52242, USA (email paul-weller@uiowa.edu) \\ *** Department of Finance, College of Business, Creighton University, \\ Omaha, NE 68178, USA (email leedunham@creighton.edu)
}

\begin{abstract}
While many technical trading rules are based upon patterns in asset prices, we lack convincing explanations of how and why these patterns arise, and why trading rules based on technical analysis are profitable. This paper provides a model that explains the success of certain trading rules that are based on patterns in past prices. We point to the importance of confirmation bias, which has been shown to play a key role in other types of decision making. Traders who acquire information and trade on the basis of that information tend to bias their interpretation of subsequent information in the direction of their original view. This produces autocorrelations and patterns of price movement that can predict future prices, such as the "headand-shoulders" and "double-top" patterns. The model also predicts that sequential price jumps for a particular stock will be positively autocorrelated. We test this prediction and find that jumps exhibit statistically and economically significant positive autocorrelations.
\end{abstract}

Keywords: technical analysis, head-and-shoulders, trading rules, confirmation bias, jump autocorrelations

\section{Introduction}

There is now convincing evidence that stock prices display short-term momentum over periods of six months to a year and longer-term mean reversion (De Bondt and Thaler, 1985; Chopra et al., 1992; Jegadeesh and Titman, 1993). There is also evidence of economically significant price reversals over short time horizons of a week to a month (Jegadeesh, 1990; Lehmann, 1990; Jegadeesh and Titman, 1995; Gutierrez and Kelley, 2008). ${ }^{1}$ This evidence provides support for trading rules designed to detect persistent trends in asset prices. Research has shown that such rules have predictive power in equity markets (Brock et al., 1992) and in foreign exchange markets (Dooley and Schafer, 1983; Sweeney, 1986; Levich and Thomas, 1993; Neely et al., 1997; Dueker and Neely, 2007).

The use of technical signals based on price patterns has received less academic attention, despite the fact that these sig- nals are widely used by practitioners (Taylor and Allen, 1992; Lui and Mole, 1998; Cheung and Chinn, 2001). At present, we lack theoretical models that can explain the presence of patternbased trading rules, though several empirical studies suggest that such rules may be profitable. Chang and Osler (1999) examine the profitability of using the "head-and-shoulders" pattern in the foreign exchange market to predict changes of trend, and find evidence of excess returns for some currencies but not others. Lo et al. (2000) develop a pattern detection algorithm based on kernel regression. They apply this methodology to identify a variety of technical price patterns including "headand-shoulders" in the US stock market over the period 19621996. They find statistical evidence that there is potentially useful information contained in most of the patterns they consider. Savin et al. (2007) show that a modified version of the algorithm of Lo et al. applied to the "head-and-shoulders" pattern

1 Conrad et al. (1991) demonstrate that bid-ask bounce explains some of this return reversal. (Cooper, 1999) and (Subrahmanyam, 2005) find that microstructure issues cannot fully explain the documented return reversal. 
has substantial predictive power for US stock returns over periods of one to three months. ${ }^{2}$

The objective of this paper is to present a theoretical model that provides an explanation for the observed autocorrelation patterns in asset returns and for the documented success of both trend-following and pattern-based technical trading rules. We do this by introducing a single cognitive bias into the model, that of confirmation bias. The bias is a phenomenon that has been extensively documented in experimental studies. It refers to the search for, or the interpretation of evidence in ways that favor existing beliefs or expectations. It has been described as "perhaps the best known and most widely accepted notion of inferential error to come out of the literature on human reasoning" (Evans, 1989, p. 41 quoted in Nickerson, 1998).

In our model, information arrival is modeled with signals of various magnitudes, arriving at differing frequencies. Large, infrequently observed signals are interpreted rationally by investors. However, investors' interpretation of less informative signals (which arrive more frequently) is biased by the recently observed large signals. The model generates price patterns, most notably the "head-and-shoulders" pattern, that have the predictive power for future stock returns claimed by technical analysts. The model thus provides a theoretical foundation for several price patterns commonly used by technical analysts. The model also produces the well-documented pattern of price momentum which can be exploited by trend-following technical rules such as those based on the comparison of short- and long-run moving averages.

In addition, our model makes several predictions. First, return autocorrelations are negative over very short horizons, positive over intermediate horizons, and become negative again over long horizons. This feature of the model conforms to the empirical properties of US equity prices described above. To our knowledge, our model is the first to simultaneously capture all three of these patterns in return autocorrelations, and provides a simple alternative to the microstructure-based explanation for negative short-horizon autocorrelations. Our model also produces a sharp prediction that the time-series of jumps in the price series should be positively autocorrelated. So far as we know, this is a new and untested empirical prediction.

We provide empirical evidence that confirms the prediction of our model that sequential price jumps in equity prices are positively autocorrelated. Specifically, we utilize the statistical bipower variation estimation technique to identify all statistically significant jumps in the daily price series of the individual component stocks of the S\&P 100 Index over the sample period 19992005. We find that sequential price jumps exhibit statistically and economically significant positive autocorrelations, and that these autocorrelations decay at a rate that is also consistent with the model.

Our model presents an alternative momentum explanation to the gradual information diffusion hypothesis of Hong and Stein (1999). In their model, newswatchers trade on fundamental information while momentum traders make trades based on past price movements. Fundamental information diffuses gradually across the newswatchers and this causes prices to underreact and display positive autocorrelation. The autocorrelation provides incentives for momentum traders whose simple trading strategies based on past prices eventually drive prices above fundamental value, leading to negative autocorrelations over longer horizons. The agents in their model are boundedly rational in that their decisions do not make use of all relevant information. Our approach is closer in spirit to that of Daniel et al. (1998) in that we assume that decisions are affected by a psychological bias. Our model setup is different and begins with the arrival of a large piece of information that is immediately and rationally impounded into prices. This news biases investors' interpretation of later information, and so in a sense is 'diffusive' in that it continues to affect future price changes. Like Hong and Stein (1999) our model predicts that certain trading strategies based on past prices can be profitable. Two notable differences are that our model predicts negative autocorrelation in the very short-run, and also explains why certain technical price patterns forecast future returns.

Our model and empirical tests also complement the recent empirical work of Gutierrez and Kelley (2008). They document negative weekly autocorrelations immediately after extreme information events, but find that momentum profits emerge several weeks after an extreme return and persist over the remainder of the year. Moreover, this momentum easily offsets the brief and initial return reversal. Our model produces predictions consistent with this finding. They also find that markets react similarly to explicit (public) and implicit (private) news, and note that many behavioral models require investors to react differently to different types of news. In contrast, our model makes no distinction between public and private news.

Zhu and Zhou (in press) offer a rather different perspective on the advantages of using technical analysis. They find that when there is uncertainty about the degree of predictability of the stock price, adding a technical Moving-Average (MA) component to the strategy that invests a fixed percentage of wealth in stocks may increase investor utility. This is because optimal dynamic strategies depend upon investors' prior beliefs and learning about unknown model-specific parameters, while MA strategies are more robust to model and parameter misspecification. ${ }^{3}$ While Zhu and Zhou focus on the effects of technical strategies on investor utility, one of our main objectives is to develop a model that captures the underlying phenomena that give rise to specific price patterns such as the head-and-shoulders or double-top patterns.

The rest of the paper is organized as follows: Sections Sections 2 and 3 present the model. Section 4 describes various trading rules and relates them to the model. In Section 5 we describe our jump-detection methodology and present empirical results. Section 6 concludes.

\section{The confirmation bias}

\subsection{Existing literature on the confirmation bias}

As noted above, the confirmation bias refers to the search for, or the interpretation of evidence in ways that favor existing beliefs or expectations. A related phenomenon has been extensively investigated in the management literature under the heading of "es-

\footnotetext{
${ }^{2}$ The value of using technical trading rules based upon past prices is still an open empirical question. Jegadeesh (2000), in his discussion of Lo et al. (2000) points out that there is no evidence of significant one-day returns after the identification of technical indicators. This finding is confirmed for the UK stock market in Dawson and Steeley (2003), while Marshall et al. (2006) find that candlestick trading strategies do not have value for Dow Jones Industrial Average Stocks. However, Savin et al. (2007) find that with longer holding periods evidence of substantial predictive power emerges. (Bessembinder and Chan, 1998) and (Allen and Karjalainen, 1999) suggest that gross profits are available from technical trading but are insufficient to cover transactions costs. Osler (2003) documents that order clustering of stop-loss and take-profit orders at round numbers provides a justification for intraday technical analysis in the currency markets. Kavajecz and Odders-White (2004) conclude that intraday technical analysis captures changes in the state of the limit order book and may add value by locating liquidity in the limit order book that allows traders to place strategic limit orders with better execution and lower transaction costs. In contrast, Marshall et al. (2008) investigate the profitability of over 5000 technical trading rules using intraday data on Standard and Poor's Depository Receipts (SPDRs) and conclude that technical analysis is not profitable after data snooping bias is taken into account.

${ }^{3}$ For a similar argument, see Blanchet-Scalliet et al. (2007).
} 
calation of commitment." This research seeks to provide explanations for commitment within organizations to losing courses of action. Theoretical explanations often focus on the theory of cognitive dissonance (Festinger, 1957). It is argued that people who are responsible for poor decisions seek to rationalize them by biasing their interpretation of information relevant for assessing the outcome of the decisions. A study of the banking industry found that bank executive turnover predicted both provisions for loan losses and the write-off of bad loans (Staw et al., 1997). The implication of these findings is that those individuals responsible for making the original loan decisions exhibited systematic bias in their interpretation of information about the status of the loans.

A specific example of how confirmation bias is recognized as a potential source of inefficiency within the investment community is provided by Camerer and Loewenstein (2004, p. 17). They report how an investment banker had described the way in which his firm combated the effects of traders' "emotional attachment to their past trades" by periodically forcing traders to switch positions with each other. In a study looking at dissonance effects in the context of mutual fund investment, Goetzmann and Peles (1997) found that even well-informed investors had a tendency to favorably distort their perceptions of the past performance of funds that they held. This may explain the observed asymmetry between investment flows into winning funds and out of losing funds (Ippolito, 1992).

Confirmation bias has also been shown to manifest itself in group decision making (Schulz-Hardt et al., 2000). Using a sample of middle managers from banks and industrial companies, the experiment involved analysis of a case study in which a company has to decide whether or not to proceed with a large investment. Subjects were required to come to a preliminary conclusion individually before being combined into groups. At this point they were given access to additional information. Groups that agreed in their preliminary conclusions showed a strong preference for accessing supporting rather than conflicting information. This finding is of particular interest in the present context, since many portfolio investment decisions are the outcome of group deliberations.

\subsection{The basic model with a single low-frequency signal}

The process by which information is revealed and incorporated into prices is constructed to capture the important features of a jump-diffusion process in a discrete-time framework. The jump-diffusion model of stock returns has a long history (Merton, 1976) and recent work by Barndorff-Nielsen and Shephard (2004) indicates that jumps in equity prices contribute a significant proportion of total price volatility. Research on empirical option pricing has also found that introducing jump components into the underlying price series alleviates some of the pricing biases found in standard models (Bates, 2003).

We suppose that there are low-frequency signals that are more informative than high-frequency signals. One can think of the low-frequency signals as generating the jumps in the price series, and the high-frequency signals as generating the diffusion. There are two groups of agents. One group is subject to cognitive bias, whereas the other is not. We assume that those subject to bias are risk neutral, but that those who are rational are risk averse. This is a simplification similar to that made by Daniel et al. (1998). It allows us to concentrate exclusively on the role of biased traders in setting prices, since it is their expectations that determine prices. Thus in what follows, all expectations will be those of the group subject to bias. The agents are endowed with shares of a risky security and of a risk free asset. They observe a low-frequency signal ( $L$-signal) at date 0 about the liquidation value of a security. At subsequent dates, a sequence of high-frequency signals $(H$-signals) is observed. At date $T$, all information about security value is revealed and the investors receive its liquidation value. The supply of the asset is fixed, and thus the price at any point in time is equal to the biased investors' expected liquidation value, given the available information.

The risky security has a liquidation value $V_{T}=\theta$, which has a prior that is normally distributed with mean zero and variance $\sigma_{\theta}^{2}$ . The signal at date 0 is

$$
L=\theta+\varepsilon,
$$

where $\varepsilon \approx N\left(0, \sigma_{\varepsilon}^{2}\right)$. It is this signal that determines the beliefs that generate subsequent confirmation bias in the risk neutral group of investors. The initial price of the asset (before the $L$-signal is observed) is determined by the prior mean of $\theta$, which is zero. Given risk neutrality, the price at date 0 is given by the expectation of $\theta$ conditional on $L$,

$$
P_{0}=E_{0}(\theta \mid L)=\frac{\sigma_{\varepsilon}^{2}}{\sigma_{\theta}^{2}+\sigma_{\varepsilon}^{2}} E(\theta)+\frac{\sigma_{\theta}^{2}}{\sigma_{\theta}^{2}+\sigma_{\varepsilon}^{2}} L=w_{0} L,
$$

where $w_{0}=\sigma_{\theta}^{2} /\left(\sigma_{\theta}^{2}+\sigma_{\varepsilon}^{2}\right)$. The first term in the expression for $P_{0}$ drops out because we have assumed the prior mean of $\theta, E(\theta)$, to be zero. The information associated with the $L$-signal is sufficiently informative that it produces a jump in the price at time $t=0$ equal to $J_{0}=w_{0} L$. The $L$-signal is followed by a sequence of $H$-signals

$$
H_{t}=\theta+\delta_{t}, \quad t=1, \ldots, T
$$

where $\delta_{t} \approx N\left(0, \sigma_{\delta}^{2}\right)$. We introduce cognitive bias into the model by making a distinction between objective and perceived signals. While the investor's interpretation of the $L$-signal is always unbiased, the L-signal determines a set of beliefs which influences the perception of the subsequent $H$-signals.

We assume that the perceived signal $\hat{H}_{t}$ takes the form

$$
\hat{H}_{t}=\theta+d\left(w_{0} L, t\right)+\delta_{t}, \quad t=1, \ldots, T .
$$

The value of the perceived $H$-signal is shifted by the value of the function $d\left(w_{0} L, t\right)$, which we call the confirmation bias function. This function is assumed to depend on: (a) the weighted $L$-signal, $w_{0} L$ and (b) the time elapsed since the $L$-signal is observed. We assume that this function takes the multiplicatively separable form

$$
d\left(w_{0} L, t\right) \equiv f\left(w_{0} L\right) m(t)
$$

The properties of $f\left(w_{0} L\right)$ are:

$$
\begin{array}{ll}
\text { P1. } & f(0)=0, \\
\text { P2. } & f^{\prime}\left(w_{0} L\right)>0, \\
\text { P3. } & f\left(-w_{0} L\right)=-f\left(w_{0} L\right)
\end{array}
$$

P1 states that when the $L$-signal is neither favorable nor unfavorable $(L=0)$ there is no subsequent bias in the perception of the $H$-signals. P2 states that when the L-signal is favorable (unfavorable), there is a positive (negative) bias in the perception of the $H$-signals, and that this bias increases with the (absolute) value of the L-signal. P3 imposes the requirement that the bias be symmetric for favorable and unfavorable signals. The properties of $m(t)$ are

$$
\begin{array}{ll}
\text { P4. } & m(t)=1, t=1 ; m(t)>0 \text { for all } t \\
\text { P5. } & \partial m / \partial t<0, t>1, \\
\text { P6. } & \lim _{t \rightarrow \infty} m(t)=0 .
\end{array}
$$

The property $\mathrm{P} 4$ of the function $m(t)$ is a straightforward normalization. P5 and P6 are intended to capture the fact that confirmation bias does not persist indefinitely, but diminishes over time, and eventually disappears. A sufficient statistic for a given sequence of objective $H$-signals is given by the average 


$$
H_{t}^{A}=\frac{1}{t} \sum_{\tau=1}^{t} H_{\tau}=\theta+\frac{1}{t} \sum_{\tau=1}^{t} \delta_{\tau} .
$$

A sufficient statistic for a given sequence of perceived $H$-signals is given by the average

$$
\hat{H}_{t}^{A}=\frac{1}{t} \sum_{\tau=1}^{t} \hat{H}_{\tau}=\theta+\frac{1}{t} f\left(w_{0} L\right) \sum_{\tau=1}^{t} m(\tau)+\frac{1}{t} \sum_{\tau=1}^{t} \delta_{\tau} .
$$

Since the bias in the perception of the public signals amounts to an additive shift in the mean, the perceived variances are not affected and are equal to the true values. From now on it is more convenient to work with precisions rather than variances, and we introduce the following notation:

$$
\pi_{\theta} \equiv \frac{1}{\sigma_{\theta}^{2}} ; \quad \pi_{\varepsilon} \equiv \frac{1}{\sigma_{\tilde{\varepsilon}}^{2}} ; \quad \pi_{\delta} \equiv \frac{1}{\sigma_{\delta}^{2}} .
$$

[equation]

The precision of $\hat{H}_{t}^{A}$ is $t \pi_{\delta}$. The equilibrium security price is given by the expectation of its liquidation value conditional on $L$ - and $H$-signals,

$$
P_{t}=w_{t}^{H} \hat{H}_{t}^{A}+w_{t} L
$$

where

$$
\begin{gathered}
w_{t}^{H} \equiv \frac{t \pi_{\delta}}{t \pi_{\delta}+\pi_{\theta}+\pi_{\varepsilon}}, \\
w_{t} \equiv \frac{\pi_{\varepsilon}}{t \pi_{\delta}+\pi_{\theta}+\pi_{\varepsilon}} .
\end{gathered}
$$

Note that the weights here are the rational Bayesian weights. Bias arises only because $\hat{H}_{t}^{A} \neq H_{t}^{A}$. This contrasts with the approach taken in behavioral models based on overconfidence, where signal precision is incorrectly perceived. The rational price is given by

$$
P_{t}^{R}=w_{t}^{H} H_{t}^{A}+w_{t} L
$$

and allows us to state the following.

\section{Proposition 1}

If investors misperceive $H$-signals, as in (3), then
(a) If $P_{0}>0$, then $P_{t}>P_{t}^{R}, t=1,2, \ldots$
(b) If $P_{0}<0$, then $P_{t}<P_{t}^{R}, t=1,2, \ldots$
(c) $\lim _{t \rightarrow \infty}\left(P_{t}-P_{t}^{R}\right)=0$

The proof is straightforward and so omitted.

The inequalities in (a) and (b) indicate that price always overreacts to the $L$-signal, but not immediately, since the immediate price jump that occurs when the L-signal arrives is always rational. Since we have normalized the initial price to zero, $P_{0}>0$ represents a (rational) positive price response generated by a favorable signal $L>0$. This is followed by subsequent prices that are greater than the fully rational price. If the signal is unfavorable, the reverse is true. Part (c) implies that the extent of the overreaction at some point starts to decline and that the asset price eventually converges to the rational price.

\subsection{Overreaction and autocorrelations}

Next we consider the evolution of price overreaction over time conditional on the realization of the $L$-signal:

$$
P_{t}-P_{t}^{R}=w_{t}^{H} \frac{f\left(w_{0} L\right)}{t} \sum_{\tau=1}^{t} m(\tau) .
$$

We need to specify functional forms for $f\left(w_{0} L\right)$ and $m(t)$, and to choose parameter values for $\pi_{\theta}, \pi_{\varepsilon}$ and $\pi_{\delta}$. We specify $f\left(w_{0} L\right)=w_{0} L$ for simplicity, and choose a (reverse) sigmoid form for $m(t)$ :

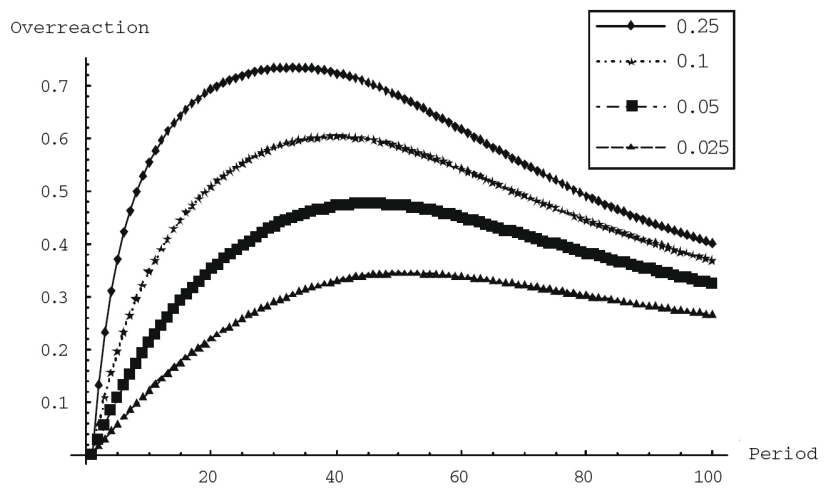

Figure 1. Price overreaction $P_{t}-P_{t}^{R}$ conditional on a favorable signal. This plots $P_{t}-P_{t}^{R}=w^{H} f\left(w_{0} L\right) / t \cdot \sum^{t} m(\tau)$ for parameter values $\pi_{\theta}=0.5, \pi_{\varepsilon}=1, L=1$, and the following form for $m(t): m(t)=1 /(1$ $+0.01 e^{0.1 t}$ ) and for different values of $\pi_{\delta}$ as indicated in the box on the right of the figure.

$$
m(t) \equiv \frac{1}{1+0.01 e^{0.1 t}} .
$$

We set $\pi_{\theta}=0.5, \pi_{\varepsilon}=L=1$, and plot $P_{t}-P_{t}^{R}$ for various values of $\pi_{\delta^{\prime}}$ the precision of the $H$-signal, in Figure 1 . The pattern of overreaction is one that initially increases, reaches a maximum and then declines. As the precision of the $H$-signals increases, the magnitude of overreaction increases. This happens because the higher precision leads to greater weight being placed on the biased perception of the $H$-signals. We examine next the pattern of return autocorrelations implied by the model. The unconditional autocorrelation function is given by

$$
\rho\left[\Delta P_{t}, \Delta P_{t+k}\right]=\frac{1}{T} \sum_{t=1}^{T-k} \frac{\operatorname{cov}\left[\Delta P_{t}, \Delta P_{t+k}\right]}{\sqrt{\operatorname{var}\left[\Delta P_{t}\right] \operatorname{var}\left[\Delta P_{t+k}\right]}} .
$$

We evaluate the autocorrelation function in the following case: $\pi_{\theta}=0.5, \pi_{\varepsilon}=1$ and $\pi_{\delta}=0.1$. The result is plotted in Figure 2 and shows a pattern of positive autocorrelations at short horizons followed by negative autocorrelations at longer horizons. It is therefore consistent with the empirical evidence documenting short-horizon momentum and long-horizon reversal. As one might expect from the results in Figure 1, qualitatively similar autocorrelation patterns emerge for other values of $\pi_{\delta}$.

The results derived from the model of this section are qualitatively the same as those obtained by Daniel et al. (1998) (henceforth DHS) from the multiperiod version of their model in which they introduce overconfidence and biased self-attribution. ${ }^{4}$ It is also true that confirmation bias has been identified as a source of overconfidence. But the way in which we model the effects of the bias is distinct from that followed by DHS. They show that biased self-attribution can generate time-varying overconfidence with respect to a private signal, and assume that public signals are correctly interpreted. But as we noted above, Gutierrez and Kelley (2008) find that markets react similarly to public and private information. We make no distinction between public and private signals, but only between the frequency (and hence the informativeness) of the signals. This turns out to generate new predictions in a model where we introduce signals of intermediate frequency. We examine this case in the next section.

\section{A model with low, medium and high-frequency signals}

The results of the basic model assume that low-frequency L-signals generate price jumps, and that these jumps are all i.i.d draws 


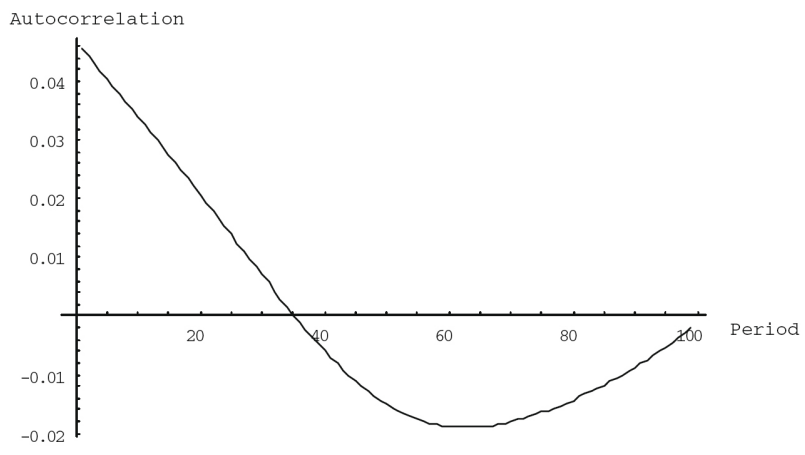

Figure 2. Unconditional return autocorrelations with a single signal. This plots the autocorrelation function $\rho\left[\Delta P_{t^{\prime}} \Delta P_{t+k}\right]=1 / T \cdot \sum_{t=k}^{1-1} \rho\left[\Delta P_{t^{\prime}}\right.$ $\left.\Delta P_{t+k}\right]$ for parameter values $\pi_{\theta}=0.5, \pi_{\varepsilon}=1$ and $\pi_{\delta}=0.1$.

from the same normal distribution. In Section 5 (below) we present empirical results suggesting that this assumption is too simple to adequately describe the process that gives rise to jumps. To preview those empirical results, when we examine high-frequency intraday returns for individual S\&P 100 stocks, we find that each stock experiences a jump (on average) once every eight days. Most of these jumps are relatively small, with an average absolute jump size of about $1.4 \%$. However, about $2 \%$ of all jumps are more than three standard deviations on either side of the mean (greater than $5 \%$ in absolute value), and occasional jumps of $10-30 \%$ are identified. Thus, jumps appear to be more consistent with a mixture of at least two normal distributions: one which generates relatively small, frequent jumps; and a second from which an occasional but very large jump occurs.

To more accurately capture these empirical jump properties in our model, we introduce a sequence of signals of intermediate frequency ( $M$-signals) that provide additional fundamental information about the value of the security. These signals are less informative and occur more frequently than the low-frequency $L$-signals. At the same time, they are informative enough to generate small price jumps, and thus are designed to correspond to the smaller, more frequent jumps just described.

\subsection{The model with $M$-signals}

We assume that the liquidation value of the security is given by

$$
V_{T}=\theta+\sum_{i=1}^{N} \lambda_{i}
$$

where $\lambda_{i} \sim N\left(0, \sigma_{\lambda}^{<}\right), \sigma_{\lambda}^{<}<\sigma_{\theta}^{<}$, and $\theta, \lambda_{i}$ are independent. The random variable $\theta$ is realized as before at time zero, and $\lambda_{i}$ is realized at time $t_{i}$.

We retain the structure of the model analyzed in the previous section, but now introduce a sequence of signals of intermediate frequency ( $M$-signals) that provide information about the new fundamental variables $\lambda_{i}$ :

$$
M_{i}=\lambda_{i}+\eta_{i} \quad i=1, \ldots, N ; \quad \eta_{i} \sim N\left(0, \sigma_{\eta}^{2}\right)
$$

occurring at time $t_{i}$. Each $M$-signal $M_{i}$ is followed by its own sequence of $H$-signals $H_{t}^{i}$ providing information about the same component of the terminal value of the asset. Thus

$$
H_{t}^{i}=\lambda_{i}+v_{t^{\prime}}^{i} \quad i=1, \ldots, N ; t=t_{i}+1, \ldots, T .
$$

where $v_{t}^{i} \sim N\left(0, \sigma_{v}^{2}\right)$. The perception of these signals is also affected by confirmation bias. Thus

$$
\begin{aligned}
& \hat{M}_{i}=\lambda_{i}+d_{M}\left(w_{0} L, t_{i}\right)+\eta_{i} \\
& \hat{H}_{t}^{i}=\lambda_{i}+d_{H}\left(w_{0} L, t_{i}\right)+v_{t}^{i}
\end{aligned}
$$

The bias functions are assumed to have the same multiplicatively separable form,

$$
d_{k}\left(w_{0} L, t_{i}\right)=a_{k} f\left(w_{0} L\right) m\left(t_{i}\right) \quad k=M, H .
$$

The constants $a_{k}$ allow us to scale the magnitude of bias according to the frequency of the signal. We assume $a_{M}>a_{H}$. Just as in the analysis of the previous section, we can represent the information contained in the public messages as

$$
\hat{H}_{t}^{A i}=\frac{1}{\left(t-t_{i}\right)} \sum_{\tau=t_{i}+1}^{t} \hat{H}_{\tau}^{i}, \quad i=1, \ldots, N ; \quad t=t_{i}+1, \ldots, T .
$$

We denote the precision of the variables $\lambda_{i^{\prime}} \eta_{i}$ and $\nu_{t}^{i}$ by $\pi_{\lambda^{\prime}} \pi_{\eta}$ and $\pi_{v^{\prime}}$ respectively. Then the asset price is given by

$$
P_{t}=\frac{t \pi_{\delta} \hat{H}_{t}^{A}+\pi_{\varepsilon} L}{t \pi_{\delta}+\pi_{\theta}+\pi_{\varepsilon}}+\sum_{i=1}^{j}\left(\frac{\left(t-t_{i}\right) \pi_{v} \hat{H}_{t}^{A i}+\pi_{\eta} \hat{M}_{i}}{\left(t-t_{i}\right) \pi_{v}+\pi_{\lambda}+\pi_{\eta}}\right), \quad t_{j}<t .
$$

The $i$ th component in the summation becomes non-zero at $t_{i^{\prime}}$ the time at which the associated $M$-signal is observed. Introducing the notation

$$
\begin{array}{ll}
w_{t-t_{i}}^{H}=\frac{\left(t-t_{i}\right) \pi_{v}}{\left(t-t_{i}\right) \pi_{v}+\pi_{i}+\pi_{\eta}}, \quad t>t_{i} ; \\
w_{t-t_{i}}^{M}=\frac{\pi_{\eta}}{\left(t-t_{i}\right) \pi_{v}+\pi_{i}+\pi_{\eta}}, \quad t \geqslant t_{i},
\end{array}
$$

we can write (20) more succinctly as

$$
P_{t}=w_{t}^{H} \hat{H}_{t}^{A}+w_{t} L+\sum_{i=1}^{j}\left(w_{t-t_{i}}^{H} \hat{H}_{t}^{A i}+w_{t-t_{i}}^{M} \hat{M}_{i}\right), \quad t_{j} \leqslant t .
$$

There are now two channels through which confirmation bias causes the observed price $P_{t}$ to diverge from its rational value. The first, as before, arises because $\hat{H}_{t}^{A} \neq H_{t}^{A} . H$-signals about $\theta$ are misperceived as a result of the bias generated by the $L$-signal. The second arises because information about the fundamental components $\lambda_{i}$ is also affected by the same source of bias.

\subsection{Effect of M-signals on autocorrelation patterns}

The first question we examine is how extending the model to incorporate signals occurring at intermediate frequency affects the pattern of return autocorrelations. We examine this with the use of numerical simulations. The parameter values chosen are as follows: $\pi_{\theta}=0.5 ; \pi_{\varepsilon}=1 ; \pi_{\delta}=0.1 ; \pi_{\lambda}=4 ; \pi_{\eta}=20 ; \pi_{v}=10 ; a_{M}=0.5$; $a_{H}=0 .{ }^{5}$ We simulate price paths over 100 periods, and assume that the time of arrival of the $M$-signals is random. The number of periods between successive signals is assumed to be lognormally distributed. It is chosen to be the exponential of a normal distribution with mean 2.3 and standard deviation 0.3 , which generates a lognormal distribution with mean 10.4 and standard deviation 3.2. So on average ten $M$-signals will occur in each simulation. The $L$ signal occurs at time 1 , and the $M$-signals that follow occur at random intervals.

The unconditional autocorrelations are plotted in Figure 3. We see that in contrast to the plot in Figure 2, at very short horizons the return autocorrelations are negative. The pattern is one of reversal followed by continuation and then again reversal. This matches the pattern of autocorrelations documented in a number of studies (Jegadeesh, 1990; Lehmann, 1990; Lo and MacKinlay, 1990; Jegadeesh and Titman, 1995; Gutierrez and Kelley, 2008).

\footnotetext{
${ }^{5}$ We choose $a_{H}=0$ for simplicity. All we require for our qualitative results is that $a_{H}$ be significantly smaller than $a_{M^{\prime}}$ i.e. bias is reduced in absolute magnitude for more frequently observed signals.
} 


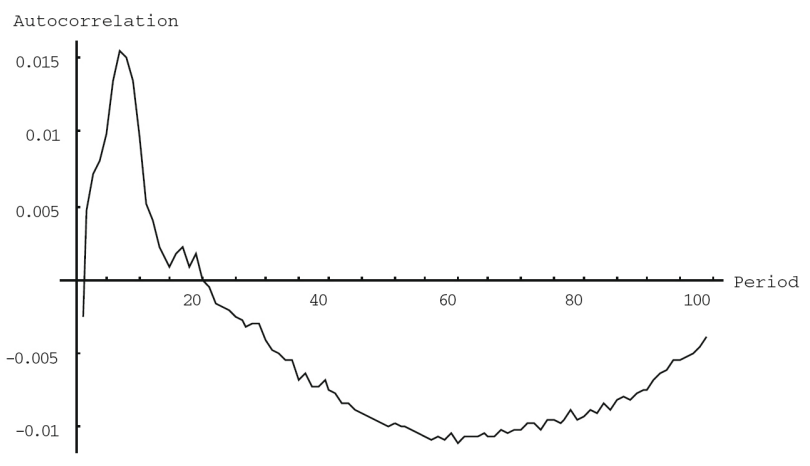

Figure 3. Autocorrelations in the model with $M$-signals. This plots unconditional autocorrelations averaged over 35,000 simulations. The parameter values are: $\pi_{\theta}=0.5, \pi_{\varepsilon}=1, \pi_{\delta}=0.1, \pi_{\lambda}=4, \pi_{\eta}=20, \pi_{v}=10$. The number of periods between $M$-signals is lognormally distributed with mean 10.43 and standard deviation 3.20, rounded to the nearest whole number.

\subsection{Further explanation of autocorrelation patterns}

In the model with $L$-signals and $H$-signals only, we observe a pattern of autocorrelations that are positive at short lags and then become negative at longer lags. However, when $M$-signals are introduced, very short lag autocorrelations are negative, become positive at intermediate lags and then again turn negative at long lags. To help understand the sources of these autocorrelation patterns, note that whenever there is a shock to an asset price that moves it away from fundamental value, this will induce negative autocorrelation at a horizon that is dependent on the speed with which the deviation is corrected.

In the model with $L$-signals and $H$-signals only, the more informative $L$-signal induces a drift away from fundamental value when the first $H$-signal is realized. This deviation from fundamental value is corrected only relatively slowly as the effects of confirmation bias dissipate. The move away from fundamental value generated by the effects of the bias causes the initial phase of positive autocorrelation. Price correction occurs relatively slowly, producing negative autocorrelations at long horizons.

$M$-signals convey additional fundamental information, but are interpreted in a biased manner, which results in a deviation from fundamental value. But learning about the fundamental underlying the $M$-signals is more rapid and at short horizons it generates negative autocorrelation that outweighs the positive autocorrelations induced by the L-signals.

In effect, the model overlays two patterns of overreaction and reversal, one over a relatively long horizon (related to the $L$-signal and its own $H$-signals) and the other over a much shorter horizon (related to each $M$-signal and its associated $H$-signals). The parameterization of the model is such that we get the initial inverted-U shape to the autocorrelation function. Clearly, the autocorrelations are dependent on the particular parameter values chosen, but they do provide an explanation for two apparently unrelated asset pricing phenomena, namely the predictive power of certain price patterns used by technical traders and the pattern of return autocorrelations. In addition, the model produces several sharp predictions about the autocorrelation in the time-series of jumps in the price series. By assumption, price jumps are associated with the occurrence of $L$-signals and $M$-signals. If we denote the $i$ th jump by $J_{i}$ we may summarize the properties of the autocorrelation in the jump series as:

\section{Proposition 2}

The correlations between jumps in the time-series (a) are positive, i.e. $\rho\left[J_{i}, J_{i+j}\right]>0$. (b) decline over time, i.e. $\rho\left[J_{i^{\prime}} J_{i+j}\right]>\rho\left[J_{i^{\prime}} J_{i+k}\right], j<k$.

(c) are increasing in the importance of the information associated with the L-signal as measured by $\sigma_{\theta}^{2}$.

(proof available upon request).

Properties (a) and (b) are self-explanatory. Property (c) makes a distinction between the importance of the information associated with the L-signal, $\sigma_{\theta}^{2}$, and the precision of the L-signal itself (captured by $\sigma_{\varepsilon}^{2}$ ). An increase in the value of $\sigma_{\theta}^{2}$ corresponds to an increased probability of larger realizations of $\theta$, and therefore a corresponding increase in the probability of larger jumps associated with the $L$-signal. Thus, larger initial jumps will lead to stronger correlations with subsequent jumps. Later in Section 5, we test the empirical predictions of Proposition 2 on jumps in US equity prices.

Although the qualitative features of our argument are not sensitive to precise parameter values, there are certain relative magnitudes that are important. The low-frequency fundamental $\theta$ is chosen to have higher variance than the higher frequency fundamental variables $\lambda_{i}$. It will have on average a larger impact on the value of the security, which then leads naturally to our assumption that it is information about the low-frequency component of fundamentals that is the source of confirmation bias. We also need to assume that signals about the higher frequency fundamental variables have higher precision so that learning about the significance of these realizations is more rapid. A framework which would render this assumption plausible is one where the low-frequency fundamental variable is concerned with information that has a high degree of ambiguity or intangibility ("soft" information) whereas the highfrequency variables relate more to "hard" information.

\section{Technical price patterns}

An important component of technical analysis is the use of price patterns as indicators of changes in a price trend. Although a large number of such indicators are used, some are regarded as more reliable than others, and are consequently more widely used by technicians. The occurrence of a particular price pattern is typically taken as an indicator of a change in a price trend, and therefore as a buy or sell signal. We will illustrate the use of such indicators in the context of two commonly used patterns. Figure 4 provides a schematic illustration of a price series displaying the "head-and-shoulders" pattern. There is fairly general agreement in books and manuals on technical analysis that the important characteristics of the head-and-shoulders pattern are the following (see, for example [Edwards and Magee, 1992; Bulkowski, 2000):

1. The "head" should be significantly taller than the "shoulders".

2. The top and bottom of the shoulders should be of roughly equal height.

3. The overall pattern should be fairly symmetric i.e. the spacing between left shoulder and head should be approximately the same as that between head and right shoulder.

The "neckline" in the figure is a straight line connecting the troughs between the two shoulders and the head. It is used to determine the point at which a trade is initiated, which is where the neckline intersects the price series after the right shoulder. The pattern signals an imminent price decline, so the technical analyst executes a short sale. Figure 5 provides an example of the headand-shoulders pattern using a daily price series from Archer Daniels Midland (ADM) during the 1997-1998 period. The left "shoulder" appears in July 1997, the "head" appears in September 1997, and the right "shoulder" appears in April 1998.

The "inverse head-and-shoulders" pattern is simply the pattern in Figure 4 viewed upside down. In this case the pattern pre- 
dicts a rise in price, and the technical analyst buys the stock. The "double-top" pattern is illustrated in Figure 6. Here the pattern is identified by the appearance of two local maxima of approximately equal value. As with head-and-shoulders, the pattern is interpreted as a signal of future price decline. The "double bottom" is an inverted double-top, and signals a future rise in price.

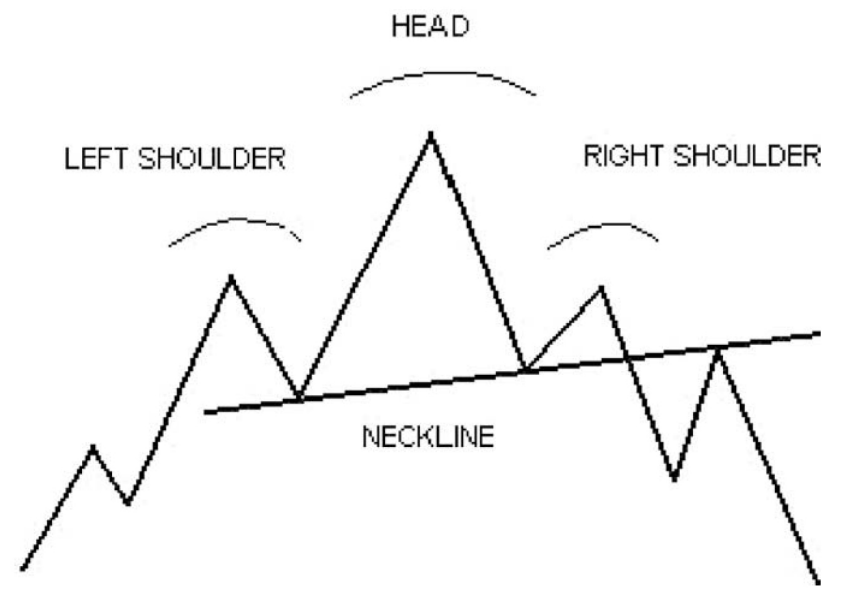

Figure 4. The head-and-shoulders pattern. This provides a schematic illustration of a price series displaying the "head-and-shoulders" pattern. The "inverse head-and-shoulders" pattern is simply the pattern in this figure viewed upside down.

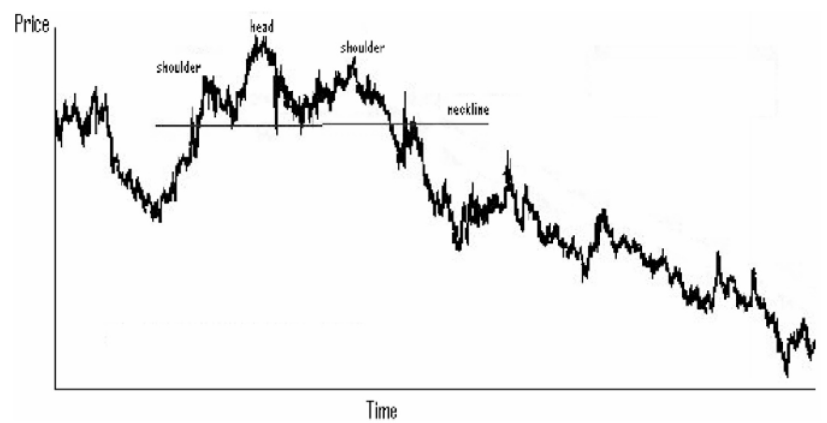

Figure 5. Head-and-shoulders pattern for Archer Daniels Midland (ADM). This provides a schematic illustration of a price series from Archer Daniels Midland (ADM) displaying the "head-and-shoulders" pattern using daily prices during 1997-1998.

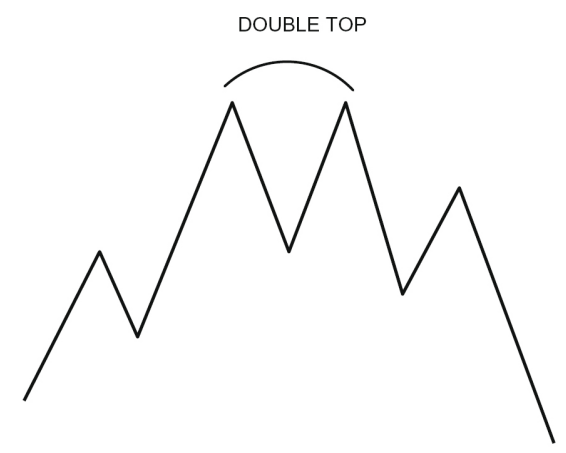

Figure 6. The double-top pattern. This illustrates the "double top" pattern. The pattern is identified by the appearance of two local maxima of approximately equal value. As with head-and-shoulders, the doubletop pattern is interpreted as a signal of future price decline. The "double bottom" is an inverted "double top", and signals a future rise in price.
There are several studies which provide evidence that price patterns contain information that may be relevant for predicting future prices. Lo et al. (2000), henceforth LMW, develop an algorithm for identifying a number of patterns including the four described above. They find that the distribution of prices conditional on a pattern occurrence is significantly different from the unconditional distribution. Savin et al. (2007) use a modified version of the LMW algorithm to show that the head-and-shoulders pattern has significant predictive power for future individual stock returns over horizons of one to three months.

An explanation for the predictive power of price patterns emerges from the model with signals of intermediate frequency. We consider the expected path of prices conditional on the initial signal $L$, which can be written as

$$
\begin{aligned}
E\left[P_{t} \mid L\right]= & w_{t} L+w_{t}^{H} E\left[\hat{H}_{t}^{A} \mid L\right]+\sum_{i=1}^{j} w_{t-t_{i}}^{\mathrm{M}} E\left[\hat{M}_{i} \mid L\right] \\
& +\sum_{i=1}^{j} w_{t-t_{i}}^{H} E\left[\hat{H}_{t}^{A i} \mid L\right] \quad t_{j} \leqslant t
\end{aligned}
$$

We represent a "typical" price pattern by examining the expected path of prices conditional on an initial $L$-signal. We use the same parameter values as for the autocorrelation plot in Figure 3, and choose $L=0.5$. However, we assume that the timing of $M$-signals is fixed and deterministic. The same pattern characteristics will be recognizable in distinct price paths even with random variation in signal timing, but if we were to average over these separate paths this would tend to obscure the features of the pattern.

Figure 7 shows a graph of the expected path of prices conditional on $L$. The head-and-shoulders pattern emerges clearly, with both shoulders and the head occurring as a result of the arrival of $M$-signals. In Figure 7, an initial L-signal has already arrived at time zero causing a positive jump (which can be inferred by noting that the expected price at time zero is positive). Subsequent $\mathrm{H}$ signals are interpreted in a biased manner, producing the upward drift. The biased interpretation and subsequent correction of the first $M$-signal produces the left shoulder; the response to the second $M$-signal generates the head, and the response to the third $M$ signal produces the right shoulder.

Thus both the head and the shoulders in the price pattern can be viewed as analogues to the broader template of momentum and reversal except that they occur at higher frequency. Momentum builds up and dissipates relatively slowly, producing the inverted $U$ price path. It results from overreaction to the low-frequency $L$-signal and is gradually corrected. On the other hand, overreaction to $M$-signals generates a sequence of inverted $\mathrm{V}$ price

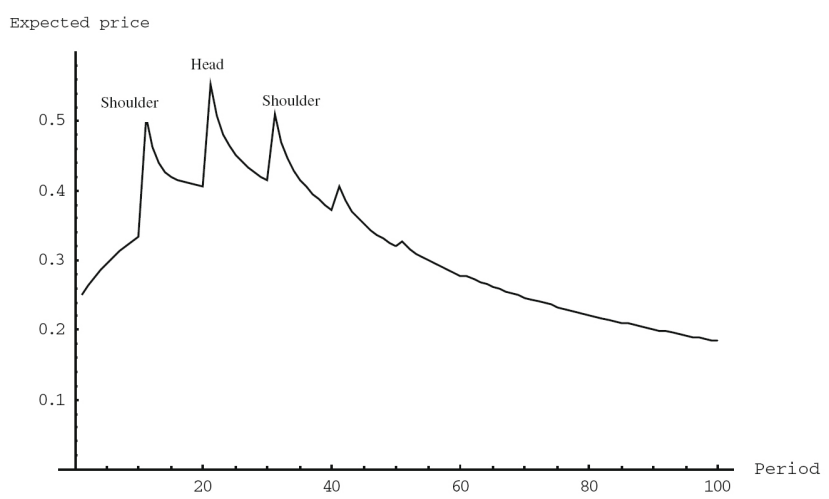

Figure 7. A "head-and-shoulders" price pattern. This plots the expected price path conditional on an initial $L$-signal $(L=0.5)$. The parameter values are the same as those used in Figure 3, except that all $M$-signals arrive at intervals of 10 periods. 


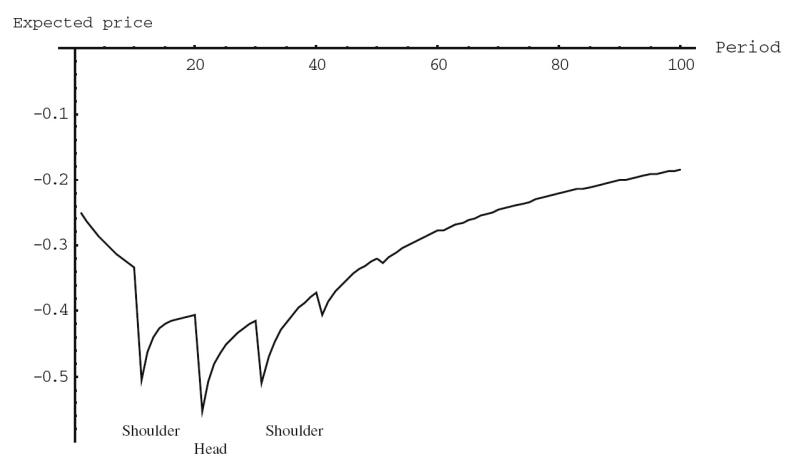

Figure 8. An inverted "head-and-shoulders" price pattern. This plots the expected price path conditional on an initial $L$-signal $(L=-0.5)$. The parameter values are the same as those used in Figure 7.

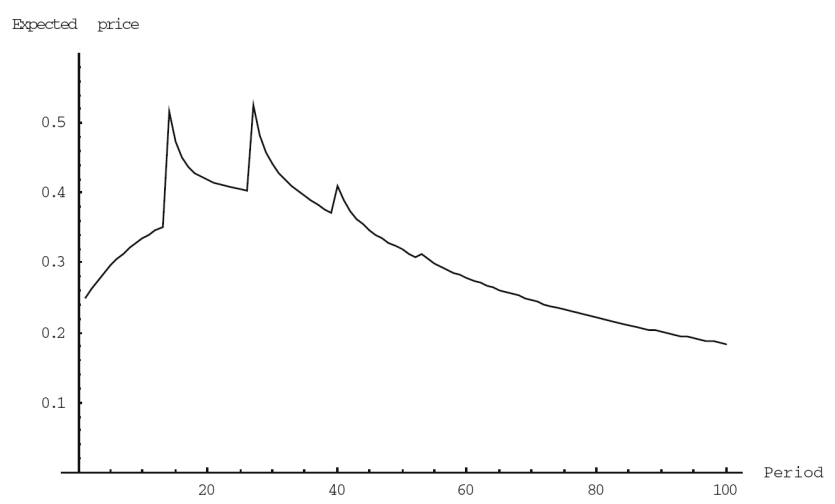

Figure 9. "Double-top" price pattern. This plots the expected price path conditional on an initial $L$-signal $(L=0.5)$. The parameter values were chosen as in Figure 3, except that all $M$-signals arrive at intervals of 13 periods.

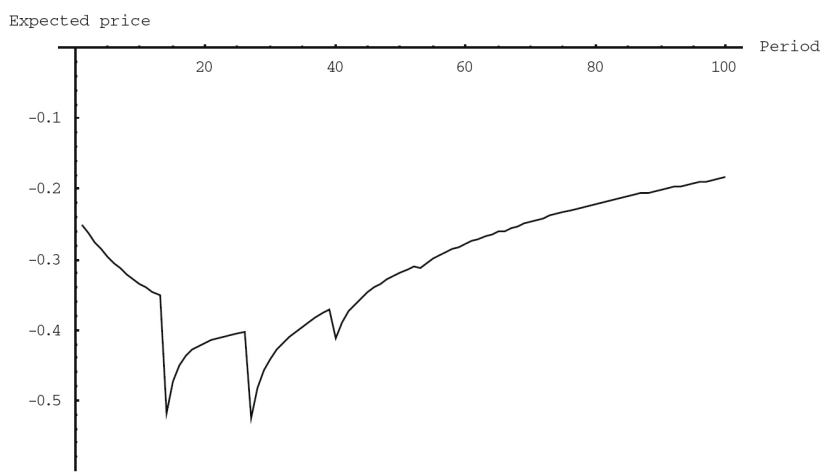

Figure 10. "Double-bottom" price pattern. This plots the expected price path conditional on an initial $L$-signal $(L=-0.5)$. The parameter values are the same as those in Figure 3, except that all $M$-signals arrive at intervals of 13 periods.

paths at higher frequency. The basic mechanism generating the inverted $\mathrm{V}$ paths is the same - overreaction followed by correction as the impact of the confirmation bias disappears. The head-andshoulders pattern emerges when one overlays the successive episodes of overreaction and correction on to the longer-term phenomenon of momentum and reversal.

The identifying features of the pattern are evident in Figure 7. In addition, it is clear that the model confirms the predictive content of the pattern and is consistent with the findings in Savin et al. (2007). The pattern appears as the momentum phase terminates and is followed by reversal. In other words, it signals an imminent price decline. Some technical analysts specify also that if the pattern is to be a reliable guide to trading it should occur after a period of significant price increase. This characteristic is also consistent with the path shown in Figure 7.

If the price path is calculated conditional on $L=-0.5$, with all other parameters given the same values, then we obtain the result illustrated in Figure 8. This pattern satisfies all the requirements for the inverted head-and-shoulders price pattern. It is a predictor of future price appreciation.

Experimentation reveals that both the head-and-shoulders and the inverted head-and-shoulders patterns are surprisingly robust, in that they will appear for different parameter values, different intervals between signals, and are also not sensitive to changes in the speed of decay captured by the function $m(t)$. This accords with the claims of technical analysts that these particular patterns are among the most reliable.

Figure 9 and Figure 10 illustrate double-top and double bottom patterns. These patterns appear when the synchronization of $M$ signals with momentum and reversal phases is shifted somewhat. Again, the price patterns predict future price movements consistent with the technical analysis literature. In summary, the price dynamics from the model with signals of intermediate frequency are consistent with the presence and validity of several of the most common technical price pattern strategies.

The explanation for the predictive power of price patterns in the model lies in the combination of momentum and reversal with price jumps that are positively correlated with the $L$-signal that generates the initial price jump and the momentum phase. Without the positive correlation resulting from confirmation bias, subsequent jumps would be equally likely to be positive or negative. A key feature of the price patterns we examine is the presence of a succession of jumps with the same sign.

For the patterns to emerge it is also necessary that prices generated by $M$-signals revert fairly rapidly to fundamental value. It is this that generates the peaks in the price series characterizing head-and-shoulders and double-top patterns. In other words, the price jumps associated with $M$-signals produce misvaluation that is quickly corrected. This in turn produces the short-horizon negative autocorrelation consistent with the findings of Gutierrez and Kelley (2008) and others.

Other researchers have proposed explanations for the phenomena of momentum and reversal in asset returns that make no use of behavioral assumptions. Lewellen and Shanken (2002) and Brav and Heaton (2002), for example, show that parameter uncertainty and learning may produce these kinds of effects. George and Hwang (2007) present evidence to suggest that long-term return reversals result from the impact of capital gain taxes. The strength of our approach lies in its ability to provide a unified framework to explain not only short-term momentum and long-term reversal, but also reversals over very short horizons and a number of commonly used technical price patterns.

\section{Jump-detection methodology and tests}

We now turn to examining the model's prediction that sequential price jumps for a particular stock will be positively autocorrelated. Recently, researchers have developed econometric techniques that can effectively separate the continuous and jump components of the underlying price process by utilizing highfrequency trade-by-trade data (Anderson et al., 2007; BarndorffNielsen and Shephard, 2004; Tauchen and Zhou, 2006). These researchers effectively demonstrate that the difference between realized volatility $(R V)$, which approximates the total daily return variance, and bi-power variation $(B V)$, which estimates the variance due to the continuous return component, is a consistent estimator of the return variance due to the jump return component. 
Jumps occur when the statistic $(R V-B V)$ is significantly different from zero, and we assume that at most one jump occurs per day.

It should be noted that we are not using the high-frequency data to examine intraday returns for the patterns described in the model. Rather, the econometric techniques require high-frequency intraday data as an input to identify when jumps occur and to estimate the size of each jump. Even though identified jumps occur almost instantaneously, the output from applying the jump-detection methodology is a time-series of daily returns, with each daily return decomposed into a continuous and jump component. These daily returns are used to examine autocorrelation patterns over longer inter-day horizons.

\subsection{Data description}

To test the predictions of the model, we are interested in obtaining a reasonably large cross-section of firms. At the same time, the data-intensity of high-frequency trade data creates practical limits on the number of firms in our sample. To balance these issues, we use the sample construction methodology of Dunham and Friesen (2007), who analyze individual stock data for the component stocks of the S\&P 100 Index as of July 1, 2006. This limits our sample to a manageable number of firms, yet captures a large percentage of the overall US equity market capitalization.

The data are collected from the NYSE's Trade and Quote (TAQ) database for the six-year sample period January 1, 1999-December 31, 2005, using only quotes from NYSE, AMEX and NASDAQ exchanges. ${ }^{6}$ Two other firms, United Postal Service and Goldman Sachs, went public during the sample period and for these firms we use data from the IPO date through the end of the sample period.

We use the bid-ask midpoint for each transaction to mitigate bid-ask bounce, and also apply several filters to eliminate erroneous observations. First, the offer/bid ratio must be less than 1.10 for the quote to be included. ${ }^{7}$ Second, we apply a sandwich filter to eliminate quotes that are $10 \%$ or further in absolute value from surrounding quotes on both sides. This filter eliminates the following erroneous type of quote sequence: a first quote that is immediately followed by a significantly higher (or lower) second quote that is subsequently followed by a third quote which is consistent with the first quote in the sequence. ${ }^{8}$ Visual inspection reveals numerous instances of such spurious quotes "sandwiched" between two otherwise consistent quotes, and our filter eliminates the erroneous quote. Without this filter, our estimation model might incorrectly identify a significant jump when prices jump to the erroneous quote and then jump back to the correct price.

We only include quotes during regular trading hours, segment each trading day into five-minute intervals and calculate interval returns using the bid-offer midpoint. For the first trading interval of each day, we utilize the opening daily bid-offer midpoint and calculate the first interval return using the bid-offer midpoint calculated at the end of the first interval. We control for stock splits by eliminating any daily interval with a five-minute return greater than $50 \%$. In short, our data set includes all of the component stocks of the S\&P 100 Index as of July 1, 2006 over the sample period 1999-2005, but excludes CBS Corporation (added to the index out of sample on January 1,2006), resulting in a final sample of 99 individual firms.

\subsection{Empirical jump properties}

We first report cross-sectional summary statistics on realized volatility and bi-power variation in Table 1 for all 99 component firms in the S\&P 100 Index over the sample period. The ratio of bi-power variation to realized volatility $(B V / R V)$, or the square root of this ratio, which can be interpreted as a standard deviation measure, has been used elsewhere in the literature to measure the fraction of total volatility generated by the continuous return component (Tauchen and Zhou, 2006). Panel (a) of Table 1 reveals that approximately $90 \%$ of total return variance for the average firm in the sample is attributable to continuous returns. Total returns are decomposed into their jump and continuous components in Panel (b) of Table 1 in the form of sample variances. ${ }^{9}$ Total risk, continuous risk and jump risk are calculated as the variance of total daily returns, variance of continuous returns, and variance of jump returns, respectively. Panel (b) of Table 1 reports that jumps contribute between $5 \%$ and $10 \%$ of the total variance in the average firm, measured as the ratio of jump risk to total risk. Note, however, that these are unconditional variance measures that include all trading days in the sample, many of which have no jump.

In Table 2, we report distributional statistics for the cross-section of equity jumps for all 99 firms in the sample, conditional upon a jump occurring. To do this, we first calculate the average value for each statistic separately for each firm in the sample. Table 2 reports the mean, median, minimum and maximum values of the firm-level averages. Panel (a) of Table 2 reports jump frequency, measured as the number of days with a jump divided by the total number of days in the sample, and shows that the average firm in the sample experiences a daily jump approximately $12 \%$ of the time, or about once every eight trading days.

Table 1. Summary statistics of equity jump data. This reports cross-sectional summary statistics for the 99 component stocks in the sample. $R V$ is the realized volatility measure and $B V$ is the bi-power variation measure. Jump returns are zero on all non-jump days, and the daily continuous return equals the total daily return minus the jump return. Total risk is the sample variance of daily returns. Continuous risk and jump risk are the sample variance of continuous and daily jump returns, respectively, using returns on all days in the sample period.

\begin{tabular}{|c|c|c|c|c|}
\hline & Mean & Median & Min & Max \\
\hline \multicolumn{5}{|c|}{ Panel (a): jump model parameters } \\
\hline$R V$ & 0.000718 & 0.000616 & 0.000285 & 0.002118 \\
\hline$R V^{1 / 2}$ & 0.023048 & 0.021965 & 0.014376 & 0.038031 \\
\hline$B V$ & 0.000649 & 0.000548 & 0.000260 & 0.001937 \\
\hline$B V^{1 / 2}$ & 0.021848 & 0.020867 & 0.013720 & 0.036493 \\
\hline$B V / R V$ & 0.9060 & 0.9087 & 0.8096 & 0.9313 \\
\hline$(B V / R V)^{1 / 2}$ & 0.9482 & 0.9498 & 0.8896 & 0.9624 \\
\hline \multicolumn{5}{|c|}{ Panel (b): risk measures } \\
\hline Total risk & 0.000442 & 0.000370 & 0.000166 & 0.001788 \\
\hline Continuous risk & 0.000406 & 0.000342 & 0.000152 & 0.001697 \\
\hline Jump risk & 0.000033 & 0.000030 & 0.000010 & 0.000132 \\
\hline Corr(jump,cont) & 0.0069 & 0.0103 & -0.2036 & 0.1398 \\
\hline
\end{tabular}

${ }^{6}$ The only S\&P 100 Index component stock not included in the study is CBS Corporation, which replaced Viacom on the S\&P 100 Index on January 1,2006, a date just outside our sample period.

${ }^{7}$ For example, on 4/07/00 American Airlines (ticker: AA) has a TAQ record consisting of a bid quote of $\$ 68.125$ and an offer quote of $\$ 80$. All other bid-offer spreads for the day were much narrower, typically less than $\$ 0.25$, and thus our filter eliminated this bid and offer quote. We also found occurrences in the TAQ quote data where a quotation appeared to be a typographical error or seemed inconsistent with surrounding quotes. For example, a bid quote of $\$ 23.375$ for HCA on $8 / 17 / 00$ is followed by a quote of $\$ 33.375$, and surrounded by bid quotes of $\$ 33$ or greater throughout the trading day.

${ }^{8}$ For example, a sequence of 3 midpoint quotes for American Airlines (ticker: AA) on 12/07/2000 are as follows; $\$ 31.03$ for interval 1, $\$ 84.91$ for interval 2, and $\$ 30.66$ for interval 3. An examination of all other quotes for AA on 12/07/2000 suggested that the $\$ 84.91$ quote for interval 2 was invalid.

${ }^{9}$ We decompose total variance, and not standard deviation, into its component parts since the variance components are additive while the standard deviation components are not. 
Table 2. Cross-sectional distributional properties of equity jumps. This reports cross-sectional summary statistics for each variable for the 99 component stocks in the S\&P 100 Index sample. Jump size is calculated as the square root of the difference between realized volatility and the bi-power variation measure. Statistics for jump size, absolute jump size and jump variance are calculated for jump days only, and thus are characteristics of the jump distribution conditional on a jump occurring. Panel (a) includes all jumps. Panel (b) includes only jumps that occur on days of high realized volatility on which an asset's realized volatility is above that asset's median realized volatility calculated over the entire sample period.

\begin{tabular}{lllll}
\hline & Mean & Median & Min & Max \\
\hline Panel (a): all jumps & & & & \\
Jump frequency & 0.120687 & 0.114204 & 0.077273 & 0.265909 \\
Jump size & -0.000272 & 0.000030 & -0.004045 & 0.004142 \\
Abs. jump size & 0.013858 & 0.013579 & 0.008939 & 0.022273 \\
Abs. daily return & 0.015698 & 0.015169 & 0.009676 & 0.025632 \\
Jump variance & 0.000275 & 0.000252 & 0.000097 & 0.000834 \\
Total variance & 0.000465 & 0.000423 & 0.000172 & 0.001149 \\
\hline Panel (b): jumps on days of high volatility & & & \\
Jump frequency & 0.122827 & 0.119318 & 0.069318 & 0.290909 \\
Jump size & -0.000411 & -0.000370 & -0.006229 & 0.005953 \\
Abs. jump size & 0.019114 & 0.018811 & 0.011627 & 0.030079 \\
Abs. daily return & 0.020887 & 0.020175 & 0.013047 & 0.036243 \\
Jump variance & 0.000467 & 0.000428 & 0.000150 & 0.001612 \\
Total variance & 0.000751 & 0.000671 & 0.000257 & 0.002531 \\
\hline
\end{tabular}

We also report an absolute jump size to provide an indication of the magnitude of jumps when they occur. Panel (a) of Table 2 reports a mean (median) absolute jump size 1.39\% (1.36\%). To shed light on the meaningfulness of this statistic, we report summary statistics on the absolute daily return in the fourth row of Panel (a). The ratio of absolute jump size to absolute daily return implies that on days when jumps occur, the jump component represents nearly $90 \%$ of the total return, on average. Lastly, Panel (a) of Table 2 also reports jump variance and total variance. The ratio of jump variance to total variance, which is analogous to the ratio $(B V / R V)$ described above, shows that jumps contribute nearly $60 \%$ of total risk for individual stocks $(0.000275 / 0.000465)$. Thus, while the unconditional contribution of jumps to returns and risk is relatively small, they account for the majority of the return and variance on the days when they do occur.

Panel (b) of Table 2 reports the same statistics as Panel (a), except that Panel (b) uses only equity jumps that occur on high realized volatility days, which we define as days where an individual stock's realized volatility is above its median realized volatility calculated over the entire sample period. As might be expected on higher volatility days, the mean (median) absolute jump size substantially increases to $1.91 \%(1.88 \%)$ but the jump frequency remains stable at around $12 \%$. While the percentage of total risk attributable to jumps is marginally higher at $62 \%$ in Panel (b), the small difference suggests that the jump contribution to total risk is fairly robust to whether or not days of low volatility are included or excluded.

\subsection{Empirical support for $M$ - and L-frequency jumps}

Table 3 reports several sets of statistics that are consistent with the presence of both $M$ - and $L$-frequency signals in the model. Panel (a) presents some basic statistics on the pooled sample of jumps which indicate that the jumps exhibit slight negative skewness and excess kurtosis. Panel (b) reports empirical quantiles for
Table 3. Pooled summary statistics and quantiles. This reports pooled distributional statistics for all significant jumps identified in our sample. Panel (a) reports basic distributional properties. Panel (b) reports actual quantiles and corresponding quantiles for a normal distribution with mean and variance equal to the empirical jump sample mean and variance. Panel (c) reports statistics for goodness-of-fit tests for the normal distribution.

Panel (a): summary statistics

Number 20,941

Mean $\quad-0.0003$

Standard Deviation $\quad 0.0171$

Skewness $\quad-0.43278$

Kurtosis 19.6313

Panel (b): jump quantiles Actual Normal Dist'n

$100 \%(\max ) \quad 0.254$

$\begin{array}{lll}99 \% & 0.0389 & 0.0394\end{array}$

$\begin{array}{lll}95 \% & 0.0241 & 0.0278\end{array}$

$\begin{array}{lll}90 \% & 0.0186 & 0.0216\end{array}$

$\begin{array}{lrr}75 \% & 0.0113 & 0.0112\end{array}$

$\begin{array}{lll}75 \% & -0.004 & -0.0003\end{array}$

$\begin{array}{lll}25 \% & -0.012 & -0.0118\end{array}$

$\begin{array}{lll}10 \% & -0.019 & -0.0221\end{array}$

$5 \%-0.024 \quad-0.0284$

$1 \%-0.038 \quad-0.0399$

$0 \%$ (Min) $\quad-0.333$

$-$

Panel (c)

Normality test

Kolmogorov-Smirnov

Cramer-von Mises

Anderson-Darling

$\begin{array}{ll}\text { Statistic } & p \text {-Value } \\ D=0.0972 & <0.01 \\ W^{2}=46.524 & <0.005 \\ A^{2}=237.812 & <0.005\end{array}$

the sample of jumps, along with corresponding quantiles for a normal distribution with mean and variance equal to the empirical jump sample mean and variance. Relative to the normal distribution, the actual jumps are more tightly clustered about the mean between the 10th and 90th percentiles, but also exhibit much longer tails than the normal distribution would predict. Panel (c) provides several formal tests that also reject the assumption of normality. While these statistics do not provide direct support for the specific form of $M$ - and $L$-signals we model, they are consistent with the notion that jumps come from at least two distributions: one which generates smaller, more frequent jumps and a second which generates occasional but very large jumps.

\subsection{Empirical autocorrelations}

We next examine the nature of the autocorrelation between sequential equity jumps. Proposition 2 indicates that sequential price jumps will be positively autocorrelated. We start by examining correlations between sequential equity jumps at time $t, t+1$, $t+2$ and $t+3$ in the first column of Table 4 . Using only those trading days on which a jump occurs, we find an average autocorrelation of nearly 0.035 between sequential price jumps. The autocorrelations between a jump at time $t$ and its subsequent three jumps are all positive and statistically significant, though the level of significance between a jump at time $t$ and $t+2$ and between a jump at time $t$ and $t+3$ is slightly weaker at $5 \%$ and $10 \%$, respectively..$^{10}$ To provide further insight, we also examine the autocorrelations on days of high realized volatility (as defined above in Table 2). These correlations are reported in the second column of Table 4, and are all positive and statistically significant at the $1 \%$ level. ${ }^{11}$

${ }^{10}$ Two potential concerns are that the positive jump correlation we document may be due either to correlated errors or investor underreaction. First, if jumps are estimated with error, then any correlation in errors may be picked up as correlation in jumps. However, this is unlikely to be driving our results since the jumps we estimate occur in a specific five-minute interval. Given that on average there are 624 five-minute intervals between jumps ( $78 \times 8$ days), any error in the initial jump estimate is more likely to be correlated with the five-minute returns that immediately follow it than with a subsequent jump. A second concern is that any time investors underreact to new information, this will lead to positive autocorrelations between the initial return and subsequent period returns. This can produce a positive correlation in jumps if both the initial and subsequent price corrections take the form of a jump. This is also unlikely to be driving our results, since it would require that the initial underreaction waits, on average, 624 periods before correcting with a second discrete jump.

${ }^{11}$ We have repeated our correlation analysis for jumps between 3 and 10 lags, and find that autocorrelations beyond 3 lags are almost uniformly positive but statistically insignificant. These estimation results are omitted for brevity but available from the authors. 
Table 4. Sequential equity jump autocorrelations. This reports correlations for sequential equity jumps at time $t, t+1, t+2$ and $t+3$. The first column includes only days where a jump occurs, which produces a sample of $n=20,840$ days; the second column includes only jumps that occur on days of high realized volatility, defined to be days on which an asset's realized volatility is above that asset's median realized volatility calculated over the entire sample period which includes $n=10,646$ days.

\begin{tabular}{lcc}
\hline & All jump days & High-volatility jump days \\
\hline Jump $t$ & 1.0000 & 1.0000 \\
Jump $t+1$ & $0.0350^{* * *}$ & $0.0384^{* * *}$ \\
Jump $t+2$ & $0.0246^{* *}$ & $0.0275^{* * *}$ \\
Jump $t+3$ & $0.0179^{*}$ & $0.0309^{* * *}$ \\
\hline
\end{tabular}

* Indicates where correlations are statistically different from zero at the $10 \%$ level. ** Indicates where correlations are statistically different from zero at the $5 \%$ level.

*** Indicates where correlations are statistically different from zero at the $1 \%$ level.

In the context of the model, jumps associated with separate $L$ frequency events are themselves uncorrelated. The predicted correlation in jumps results from the jumps associated with $M$-frequency signals that follow a particular $L$-frequency event. Thus, the correlations reported in Table 4 can be refined by identifying $L$-frequency jumps, and estimating correlations only between the L-jumps and the jumps immediately following them. Because our empirical jump-detection methodology simply identifies significant jumps, it cannot explicitly identify $L$-frequency jumps. However, in the model $L$-frequency jumps are larger in magnitude than $M$-frequency jumps, and Proposition 2 indicates that the larger the value of the $L$-frequency jumps (as captured by $\sigma_{\theta}^{2}$ ), the stronger the correlation with subsequent $M$-frequency jumps.

Thus, we sort each firm's jumps into deciles based upon the absolute jump size, and use the jumps in the largest one or two deciles as a proxy for that firm's L-frequency jumps. Panel (a) of Table 5 reports cross-sectional statistics for each decile of size-sorted jumps, and Panels (b) and (c) report correlations between $L$-frequency jumps and the jumps that follow them. In Panel (b), $L$-frequency jumps are identified as jumps in the largest two deciles, while in Panel (c) we use only the largest decile of absolute jumps. Conditioning on $L$-frequency jumps in this manner, we find that the correlations are two to three times larger than those reported in Table 4. In particular, the lag- 1 correlation is 0.0603 in Panel (b) and 0.0919 in Panel (c), and both correlations are statistically significant at the $1 \%$ level.

While these correlations are small in absolute value, the size of the observed correlation in jumps depends upon the magnitude of the underlying investor bias. Few studies have explicitly quantified the magnitude of the confirmation bias in individuals, although Friesen and Weller (2006) measure the magnitude of the closely related cognitive dissonance bias in financial analyst forecasts. They find that while the cognitive dissonance bias is present, it is relatively small in magnitude. Specifically, their estimates suggest that cognitive dissonance introduces a mean-shift bias into analyst forecasts of between $5 \%$ and $10 \%$ of the lagged forecast error. In light of their finding, the correlations of 0.06 and 0.09 reported in Panels (b) and (c) of Table 5 seem reasonable.

\section{Conclusion}

This paper develops a theoretical framework that can account for the apparent success of both trend-following and patternbased technical trading rules. Our model introduces a single cognitive bias, which has been extensively documented in the psychological literature and describes the tendency of individuals to search for and interpret information selectively to conform to a given set of beliefs.

In the model, information arrival is modeled with signals of various magnitudes, arriving at differing frequencies. Large, infrequently observed signals are interpreted rationally by investors. However, investors' interpretation of less informative signals
Table 5. Autocorrelations between L-frequency jumps and sequential jumps. For each firm, significant jumps are sorted into deciles based on absolute jump size. Panel (a) reports cross-sectional statistics on size-sorted jumps. Thus, the smallest $10 \%$ of jumps for each firm are pooled into decile 1 and the largest $10 \%$ of jumps for each firm are in decile 10 . For this reason, the max jump size in decile $\mathrm{j}$ can be larger than the min jump size in decile $j+1$. Panel (b) reports correlations for $L$-frequency jumps and sequential equity jumps at time $t, t+1, t+2$ and $t+3$, where $L$-frequency jumps are identified as jumps in the largest two deciles. Panel (c) reports similar correlations, but identifies $L$-frequency jumps as those in the top decile only.

\begin{tabular}{lcccc}
\hline Panel (a): absolute jump size & & & \\
& Mean & Median & Min & Max \\
\hline Decile & & & & \\
1 (smallest) & 0.00526 & 0.00510 & 0.00197 & 0.01089 \\
2 & 0.00706 & 0.00681 & 0.00384 & 0.01299 \\
3 & 0.00840 & 0.00813 & 0.00508 & 0.01572 \\
4 & 0.00967 & 0.00936 & 0.00611 & 0.01776 \\
5 & 0.01099 & 0.01072 & 0.00716 & 0.02068 \\
6 & 0.01251 & 0.01221 & 0.00810 & 0.02296 \\
7 & 0.01448 & 0.01408 & 0.00904 & 0.02624 \\
8 & 0.01706 & 0.01659 & 0.01001 & 0.03204 \\
9 & 0.02095 & 0.02038 & 0.01091 & 0.03810 \\
10 (largest) & 0.03317 & 0.02976 & 0.01438 & 0.33337 \\
\end{tabular}

Panel (b): correlations between L-frequency jumps and subsequent jumps (deciles 9 and 10;

$n=4150) \quad \begin{array}{lllll}t & t+1 & t+2 & t+3\end{array}$

Jump $t \quad 1.0000$

Jump $t+1 \quad 0.0603 * * * \quad 1.0000$

Jump $t+2 \quad 0.0400 * * * \quad 0.0403 * * * \quad 1.0000$

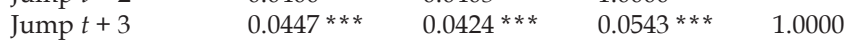

Panel (c): correlations between L-frequency jumps and

subsequent jumps (decile 10;

$\begin{array}{lllll}n=2053) & t & t+1 & t+2 & t+3 \\ \text { Jump } t & 1.0000 & & & \\ \text { Jump } t+1 & 0.0919 * * & 1.0000 & & \\ \text { Jump } t+2 & 0.0297 & 0.0380 * & 1.0000 & \\ \text { Jump } t+3 & 0.0397 * & 0.0304 & 0.0738 * * * & 1.0000\end{array}$

* Indicates where correlations are statistically different from zero at the $5 \%$ level. ** Indicates where correlations are statistically different from zero at the $10 \%$ level. *** Indicates where correlations are statistically different from zero at the $1 \%$ level.

(which arrive more frequently) is biased by the recently observed large signals. The model generates price patterns that conform to a number of well-documented trading strategies.

In addition, our model makes two empirically testable predictions. First, return autocorrelations are negative over very short horizons, positive over intermediate horizons, and become negative again over long horizons. This feature of the model conforms to the well-documented empirical properties of US equity prices. Our model also predicts that the time-series of jumps in the price series should be positively autocorrelated. We provide empirical evidence that confirms the prediction of our model that the sequential price jumps in equity prices are positively autocorrelated. Specifically, we utilized the bi-power variation estimation technique described in Tauchen and Zhou (2006) to identify all statistically significant equity jumps on the individual component stocks of the S\&P 100 Index over the sample period 1999-2005. We find that sequential equity jumps exhibit statistically and economically significant positive autocorrelations.

Our model and empirical tests complement the recent empirical work of Gutierrez and Kelley (2008), who document negative weekly autocorrelations immediately after extreme information events, but find that momentum profits emerge several weeks after an extreme return and persist over the remainder of the year. This finding is consistent with the predictions of our model. Also consistent with our model, they find that markets react similarly to explicit (public) and implicit (private) news. 


\section{References}

Allen and Karjalainen, 1999 - F. Allen and R. Karjalainen, Using genetic algorithms to find technical trading rules, Journal of Financial Economics 51 (1999), pp. 245-271.

Anderson et al., 2007 - T. Anderson, T. Bollerslev and F. Diebold, Roughing it up: Including jump components in the measurement, modeling and forecasting of return volatility, Review of Economics and Statistics 89 (2007), pp. 701-720.

Barndorff-Nielsen and Shephard, 2004 - O. Barndorff-Nielsen and N. Shephard, Power and bi-power variation with stochastic volatility and jumps, Journal of Financial Econometrics 2 (2004), pp. 1-48.

Bates, 2003 - D. Bates, Empirical option pricing: A retrospection, Journal of Econometrics 116 (2003), pp. 387-404.

Bessembinder and Chan, $1998-\mathrm{H}$. Bessembinder and K. Chan, Market efficiency and the returns to technical analysis, Financial Management 27 (1998), pp. 5-13.

Blanchet-Scalliet et al., 2007 - C. Blanchet-Scalliet, A. Diop, R. Gibson, D. Talay and E. Tanré, Technical analysis compared to mathematical models based methods under parameters misspecification, Journal of Banking and Finance 31 (2007), pp. 1351-1373.

Brav and Heaton, 2002 - A. Brav and J.B. Heaton, Competing theories of financial anomalies, Review of Financial Studies 15 (2002), pp. 575-606.

Brock et al., 1992 - W. Brock, J. Lakonishok and B. LeBaron, Simple technical trading rules and the stochastic properties of stock returns, Journal of Finance 47 (1992), pp. 1731-1764.

Bulkowski, 2000 - T. Bulkowski, Encyclopedia of Chart Patterns, John Wiley and Sons, New York (2000).

Camerer and Loewenstein, 2004 - C.F. Camerer and G. Loewenstein, Behavioral economics: Past present and future. In: C.F. Camerer, G. Loewenstein and M. Rabin, Editors, Advances in Behavioral Economics, Princeton University Press, New Jersey (2004).

Chang and Osler, 1999 - P.H.K. Chang and C. Osler, Methodical madness: Technical analysis and the irrationality of exchange rate forecasts, Economic Journal 109 (1999), pp. 636-652.

Cheung and Chinn, 2001 - Y. Cheung and M. Chinn, Currency traders and exchange rate dynamics: A survey of the US market, Journal of International Money and Finance 20 (2001), pp. 439-471.

Chopra et al., 1992 - N. Chopra, J. Lakonishok and J. Ritter, Measuring abnormal performance: Do stocks overreact?, Journal of Financial Economics $\mathbf{3 1}$ (1992), pp. 235-268.

Chuang and Lee, 2006 - W. Chuang and B. Lee, An empirical evaluation of the overconfidence hypothesis, Journal of Banking and Finance 30 (2006), pp. 2489-2515.

Conrad et al., 1991 - J. Conrad, G. Kaul and M. Nimalendran, Components of short-horizon individual security returns, Journal of Financial Economics 29 (1991), pp. 365-384.

Cooper, 1999 - M. Cooper, Filter rules based on price and volume in individual security overreaction, Review of Financial Studies 12 (1999), pp. 901-935.

Daniel et al., 1998 - K. Daniel, D. Hirshleifer and A. Subrahmanyam, Investor psychology and security market under- and over-reactions, Journal of Finance 53 (1998), pp. 1839-1885.

Dawson and Steeley, 2003 - E. Dawson and J. Steeley, On the existence of visual technical patterns in the UK stock market, Journal of Business Finance and Accounting 30 (2003), pp. 263-293.

De Bondt and Thaler, 1985 - W. De Bondt and R. Thaler, Does the stock market overreact?, Journal of Finance 40 (1985), pp. 793-805.

Dooley and Schafer, 1983 - M. Dooley and J. Schafer, Analysis of short-run exchange rate behavior: March 1973 to November 1981. In: D. Bigman and T. Taya, Editors, Exchange Rate and Trade Instability: Causes Consequences and Remedies, Ballinger, Cambridge (1983).

Dueker and Neely, 2007 - M. Dueker and C.J. Neely, Can Markov switching rules predict foreign exchange returns?, Journal of Banking and Finance 31 (2007), pp. 279-296.

Dunham and Friesen, 2007 - L. Dunham and G. Friesen, An empirical examination of jump risk in US equity and bond markets, North American Actuarial Journal 11 (2007), pp. 76-91.

Edwards and Magee, 1992 - R. Edwards and J. Magee, Technical Analysis of Stock Trends, New York Institute of Finance, Business Information and Publishing, New York (1992).

Evans, 1989 - J. Evans, Bias in Human Reasoning: Causes and Consequences, Erlbaum, New Jersey (1989).

Festinger, 1957 - L. Festinger, A Theory of Cognitive Dissonance, Stanford University Press, California (1957).

Friesen and Weller, 2006 - G. Friesen and P. Weller, Quantifying cognitive biases in analyst earnings forecasts, Journal of Financial Markets 9 (2006), pp. 333-365.

George and Hwang, 2007 - T. George and C. Hwang, Long-term reversals: Overreaction or taxes?, Journal of Finance 62 (2007), pp. 2865-2896.
Goetzmann and Peles, 1997 - W. Goetzmann and N. Peles, Cognitive dissonance and mutual fund investors, Journal of Financial Research 20 (1997), pp. 145-158.

Gutierrez and Kelley, 2008 - R. Gutierrez Jr. and E. Kelley, The long-lasting momentum in weekly returns, Journal of Finance 63 (2008), pp. 415-447.

Hong and Stein, $1999-$ H. Hong and J. Stein, A unified theory of underreaction, momentum trading and overreaction in asset markets, Journal of Finance 54 (1999), pp. 2143-2184.

Ippolito, 1992 - R. Ippolito, Consumer reaction to measures of poor quality: Evidence from the mutual fund industry, Journal of Law and Economics 35 (1992), pp. 45-70.

Jegadeesh, 1990 - N. Jegadeesh, Evidence of predictable behavior of security returns, Journal of Finance 45 (1990), pp. 881-898.

Jegadeesh, 2000 - N. Jegadeesh, Discussion of foundations of technical analysis, Journal of Finance 55 (2000), pp. 1765-1770.

Jegadeesh and Titman, 1993 - N. Jegadeesh and S. Titman, Returns to buying winners and selling losers: Implications for stock market efficiency, Journal of Finance 48 (1993), pp. 65-91.

Jegadeesh and Titman, 1995 - N. Jegadeesh and S. Titman, Overreaction, delayed reaction and contrarian profits, Review of Financial Studies 8 (1995), pp. 973-993.

Kavajecz and Odders-White, 2004 - K. Kavajecz and E. Odders-White, Technical analysis and liquidity provision, Review of Financial Studies 17 (2004), pp. 1043-1071.

Lehmann, 1990 - B. Lehmann, Fads, martingales and market efficiency, Quarterly Journal of Economics 105 (1990), pp. 1-28.

Levich and Thomas, 1993 - R. Levich and L. Thomas, The significance of technical trading rule profits in the foreign exchange market: A bootstrap approach, Journal of International Money and Finance 12 (1993), pp. 451-474.

Lewellen and Shanken, 2002 - J. Lewellen and J. Shanken, Learning, asset-pricing tests, and market efficiency, Journal of Finance 57 (2002), pp. 1113-1145.

Lo and MacKinlay, 1990 - A. Lo and A.C. MacKinlay, When are contrarian profits due to stock market overreaction?, Review of Financial Studies 3 (1990), pp. 175-205.

Lo et al., 2000 - A. Lo, H. Mamaysky and J. Wang, Foundations of technical analysis: Computational algorithms, statistical inference and empirical implementation, Journal of Finance 55 (2000), pp. 1705-1765.

Lui and Mole, 1998 - Y. Lui and D. Mole, The use of fundamental and technical analyses by foreign exchange dealers: Hong Kong evidence, Journal of International Money and Finance 17 (1998), pp. 535-545.

Marshall et al., 2006 - B. Marshall, M. Young and L. Rose, Candlestick technical trading strategies: Can they create value for investors?, Journal of Banking and Finance 30 (8) (2006), pp. 2303-2323.

Marshall et al., 2008 - B. Marshall, R. Cahan and J. Cahan, Does intraday technical analysis in the US equity market have value?, Journal of Empirical Finance 15 (2008), pp. 199-210.

Merton, 1976 - R. Merton, Option pricing when underlying stock returns are discontinuous, Journal of Financial Economics 3 (1976), pp. 125-144.

Neely et al., 1997 - C.J. Neely, P. Weller and R. Dittmar, Is technical analysis in the foreign exchange market profitable? A genetic programming approach, Journal of Financial and Quantitative Analysis 32 (1997), pp. 405-426.

Nickerson, 1998 - R. Nickerson, Confirmation bias: A ubiquitous phenomenon in many guises, Review of General Psychology 2 (1998), pp. 175-220.

Osler, 2003 - C. Osler, Currency orders and exchange rate dynamics: An explanation for the predictive success of technical analysis, Journal of Finance 58 (2003), pp. 1791-1820.

Savin et al., 2007 - N.E. Savin, P. Weller and J. Zvingelis, The predictive power of 'head and shoulders' price patterns in the US stock market, Journal of Financial Econometrics 5 (2007), pp. 243-265.

Schulz-Hardt et al., 2000 - Stefan Schulz-Hardt, Dieter Frey, Carsten Lüthgens and Serge Moscovici, Biased information search in group decision making, Journal of Personality and Social Psychology 78 (2000), pp. 655-669.

Staw et al., 1997 - B. Staw, S. Barsade and K. Koput, Escalation at the credit window: A longitudinal study of bank executives' recognition and write-off of problem loans, Journal of Applied Psychology 82 (1997), pp. 130-142.

Subrahmanyam, 2005 - A. Subrahmanyam, Distinguishing between rationales for short-horizon predictability in stock returns, Financial Review 40 (2005), pp. 11-35.

Sweeney, 1986 - R. Sweeney, Beating the foreign exchange market, Journal of Finance 41 (1986), pp. 163-182.

Tauchen and Zhou, 2006 - Tauchen, G., Zhou, H., 2006. Realized jumps on financial markets and predicting credit spreads. Finance and Economics Discussion Series 2006-35, Board of Governors of the Federal Reserve System.

Taylor and Allen, 1992 - M. Taylor and H. Allen, The use of technical analysis in the foreign exchange market, Journal of International Money and Finance 11 (1992), pp. 304-314.

Zhu and Zhou, in press - Y. Zhu and Guofu Zhou, Technical analysis: An asset allocation perspective on the use of moving averages. Journal of Financial Economics (in press). 